\title{
Turbulence comes in bursts in stably stratified flows
}

\author{
C. Rorai, ${ }^{1,2}$ P. D. Mininni, ${ }^{3}$ and A. Pouquet ${ }^{4}$ \\ ${ }^{1}$ Nordita, Roslagstullsbacken 23, 10691 Stockholm, Sweden \\ ${ }^{2}$ ICTP, Strada Costiera 11,34151 Trieste, Italy \\ ${ }^{3}$ Departamento de Física, Facultad de Ciencias Exactas y Naturales, Universidad de Buenos Aires, C1053ABJ Buenos Aires, Argentina \\ and IFIBA, CONICET, Ciudad Universitaria, 1428 Buenos Aires, Argentina \\ ${ }^{4}$ Laboratory for Atmospheric and Space Physics, University of Colorado Boulder, Boulder, Colorado 80309, USA
}

(Received 13 April 2013; revised manuscript received 10 February 2014; published 2 April 2014)

\begin{abstract}
There is a clear distinction between simple laminar and complex turbulent fluids; however, in some cases, as for the nocturnal planetary boundary layer, a stable and well-ordered flow can develop intense and sporadic bursts of turbulent activity that disappear slowly in time. This phenomenon is ill understood and poorly modeled and yet it is central to our understanding of weather and climate dynamics. We present here data from direct numerical simulations of stratified turbulence on grids of $2048^{3}$ points that display the somewhat paradoxical result of measurably stronger events for more stable flows, not only in the temperature and vertical velocity derivatives as commonplace in turbulence, but also in the amplitude of the fields themselves, contrary to what happens for homogenous isotropic turbulent flows. A flow visualization suggests that the extreme values take place in Kelvin-Helmoltz overturning events and fronts that develop in the field variables. These results are confirmed by the analysis of a simple model that we present. The model takes into consideration only the vertical velocity and temperature fluctuations and their vertical derivatives. It indicates that in stably stratified turbulence, the stronger bursts can occur when the flow is expected to be more stable. The bursts are generated by a rapid nonlinear amplification of energy stored in waves and are associated with energetic interchanges between vertical velocity and temperature (or density) fluctuations in a range of parameters corresponding to the well-known saturation regime of stratified turbulence.
\end{abstract}

DOI: 10.1103/PhysRevE.89.043002

\section{INTRODUCTION}

Large fluctuations are common in physical systems with long-range correlations and have been found to be linked to so-called $1 / f$ noise [1]. They take the form of sporadic and localized events, as observed in many instances in critical phenomena and in turbulent flows, and are diagnosed through non-Gaussian probability distribution functions (PDFs) [2]. In turbulence, strong events occur in field gradients such as the vorticity field or in the presence of fronts in passive tracers such as chemical pollutants, with the velocity itself being nearly Gaussian. There are however exceptions to this last rule for shear flows [3], quantum fluids [4,5], and subtropical current systems [6]. In all three cases, the velocity itself is observed to develop strong values that are responsible for the non-Gaussian wings of their PDFs. Also in the mesosphere and lower troposphere [7] and in the solar wind [8,9], the velocity field and the density were observed to be non-Gaussian in the presence of large-scale fronts.

The occurrence of strong events in field gradients, the so-called internal intermittency, is viewed as a signature of fully developed turbulence and is therefore more surprising in stable flows. Internal intermittency is often associated with spatially localized coherent structures such as vortex filaments in hydrodynamics or current sheets in magnetohydrodynamics. Intermittency can also be associated with the transition in a flow to the turbulent regime $[10,11]$.

Intermittency makes the stable nocturnal planetary boundary layer (PBL) highly unpredictable: As night sets in, this layer between the atmosphere and land or sea stabilizes due to the radiative cooling of the ground and of the ocean masses. It is still unclear how stable the nocturnal PBL becomes. Three regimes have been observed [12]: very stable, weakly stable
PACS number(s): 47.55.Hd, 47.27.- $\mathrm{i}$, 47.35.Bb

with turbulent motions persisting and competing with internal gravity waves, and transitory. Even in the very stable case, the PBL is subject to intense sporadic bursts of turbulence that die out after many wave periods $[12,13]$.

Numerical simulations play an increasing role in the understanding of these complex processes and in quantifying the dual problem of the increased stability [14] and the spontaneous generation of bursts. However, modeling of the PBL in weather and climate codes is often inadequate, resulting, for example, in an inaccurate evaluation of the extension of the ice sheet, as is the case over Greenland [15], and in a faulty estimate of the overall energy balance in long-term climate systems. Indeed, the PBL affects, for example, mixing, frost occurrence, aerosol dispersion, and air quality [16].

The complex behavior of geophysical flows can be attributed to a variety of external factors, including moisture in the atmosphere, salinity in the oceans, and boundaries. However, the question remains as to what the simplest system is that can display such a behavior. This is the approach we take in this paper, using large-scale direct numerical simulations and modeling in the framework of the Boussinesq system in which the potential temperature (or density) variations enter only in the buoyancy force, as the flow remains otherwise incompressible.

\section{EQUATIONS AND MODELS}

In this section we introduce the Boussinesq equations (which are later solved explicitly in the direct numerical simulations) and derive from these equations a very simple model for the evolution of field variations in a stratified flow. 


\section{A. Boussinesq equations}

We start from the Boussinesq equations, which describe a stably stratified flow with gravity in the vertical direction. For the velocity $\mathbf{u}=(u, v, w)$ and potential temperature fluctuations $\theta$, the equations are

$$
\begin{aligned}
\frac{\partial \mathbf{u}}{\partial t}+\mathbf{u} \cdot \nabla \mathbf{u} & =-\nabla P-N \theta e_{z}+v \Delta \mathbf{u}+\mathbf{f}_{V}, \\
\frac{\partial \theta}{\partial t}+\mathbf{u} \cdot \nabla \theta & =N w+\kappa \Delta \theta, \\
\nabla \cdot \mathbf{u} & =\mathbf{0},
\end{aligned}
$$

where $P$ is the pressure, $\kappa=v$ the diffusivity, and $\mathbf{f}_{V}$ a velocity forcing term. The square Brunt-Väisälä frequency is given by $N^{2}=-(g / \theta)(d \bar{\theta} / d z)$, where $\bar{\theta}$ is the imposed background stratification, assumed to be linear, and $g$ is the gravity.

\section{B. Simple model}

We are interested in a simple model for the evolution of the field variations. Estimating the pressure forces in Eq. (1), which for an incompressible fluid are highly nonlocal, is difficult since one has to consider the coupling between vorticity and shear. A simple one-dimensional model of such behavior was developed in Ref. [17]. This model, sometimes called restricted Euler dynamics, has proven useful in analyzing the development and the statistical and geometrical properties of intermittent structures in a variety of turbulent flows (see [18] for a recent review).

For simplicity, in the absence of stratification one can consider only vertical velocity differences $\delta w$ in the vertical velocity $w$ at scale $\ell$, defined as $\delta w(\ell)=\langle w(\mathbf{x}+\ell \hat{z})-$ $w(\mathbf{x})\rangle \approx \ell \partial_{z} w$. Taking the spatial derivative of Eq. (1) in the one-dimensional (1D) case, with $\theta=0$, and neglecting pressure, forcing, and viscous forces yields

$$
\partial_{t}\left(\partial_{z} w\right)+w \partial_{z}\left(\partial_{z} w\right)=d_{t}\left(\partial_{z} w\right)=-\left(\partial_{z} w\right)^{2} .
$$

Then, for the velocity differences $d_{t} \delta w=-\delta w^{2} / \ell$, this equation immediately shows the temporal enhancement of negative values of $\delta w$, as observed, for example, for isotropic turbulent fluids for which the skewness of velocity gradients is negative and of order unity.

When the flow is stably stratified, gravity acts as a restitutive force allowing for oscillatory solutions (internal gravity waves). Nonlinear coupling tends to transfer energy towards modes with vertical spatial dependence, resulting in the creation of horizontal layers in the fluid, and further justifying the reduction to a $1 \mathrm{D}$ system. Under the same hypothesis, for $\delta \theta \approx \ell \partial_{z} \theta$, and from Eqs. (1) and (2) we obtain

$$
\begin{aligned}
& \frac{d \delta w}{d t}=-\frac{\delta w^{2}}{\ell}-N \delta \theta, \\
& \frac{d \delta \theta}{d t}=-\frac{\delta w \delta \theta}{\ell}+N \delta w .
\end{aligned}
$$

These equations can be considered as a crude 1D (vertical) model of a stratified flow.

We can define the dimensionless Froude number $\mathrm{Fr}=$ $U / N L$ (with $U$ and $L$ characteristic velocity and length); it quantifies the ratio between nonlinear and linear effects.



FIG. 1. Evolution in time of vertical velocity variations $\delta w$ in the model of Eqs. (4) and (5) for $\ell=0.2$ and $N=0$ (no stratification, solid line), 2 (dotted line), 4 (dashed line), 12 (dash-dotted line), 20 (dash-triple-dotted line), and 30 (long-dashed line). Note the faster evolution towards negative and strong vertical gradients at intermediate values of $N$, as it increases from 2 to 12 , before oscillatory behavior takes over for large enough $N$ (here, corresponding to $N=20$ and 30).

The system of Eqs. (4) and (5) has only one fixed point ( $\delta w=\delta \theta=0)$. For weak stratification (large Froude number), one recovers the Euler behavior of strong negative gradients and in the opposite case ( $N \gg 1$, or small Froude number), the model has oscillatory solutions in the vertical velocity and temperature fluctuations (see Fig. 1).

The terms governing both (nonlinear and linear) behaviors become comparable when $\delta w \sim \delta \theta \sim N \ell$. When this is satisfied in a range of scales, it corresponds to the balanced energy spectrum $E\left(k_{z}\right) \sim \delta w^{2} / k_{z} \sim N^{2} k_{z}^{-3}$, which has been predicted and observed in many instances in the atmosphere and the oceans (see, e.g., Ref. [19] and Fig. 2).

In Fig. 1 an interesting evolution is observed. In an intermediate regime (specifically, here $\ell=0.2$ and $N=2$, 4 , and 12) and for initial $\delta w$ and $\delta \theta>0, \delta w$ becomes negative (and diverges) unlike the case $N=0$ and it does so faster for larger values of $N$. In other words, the effect of waves is rapidly amplified by the nonlinear term, resulting in a catastrophic behavior. The runaway occurs as $N$ increases and before oscillations take over, in Fig. 1 for $N>12$. Note that, for $N=0$ and initial $\delta w<0$, large negative gradients do eventually occur: This is the essence of the Vieillefosse model $[17,18]$, written to study the development of strong negative gradients in homogeneous isotropic turbulence. However, they do so more slowly than in the presence of gravity waves, as shown in Fig. 1.

The large negative values of $\delta w$ can be interpreted as the signature of strong intermittent bursts. Note that for even larger values of $N$, although the solutions become oscillatory, they still display skewness (i.e., they have a tendency towards more negative values of $\delta w)$. On the other hand, if the initial conditions are negative $(\delta w, \delta \theta<0)$, the divergence is delayed by increasing stratification.

The coupling of this evolution to that of the horizontal velocity damps the runaway evolution of $\delta w$ (because of incompressibility) but strong gradients still form. For such extensions, see Ref. [20], which presents a similar model 

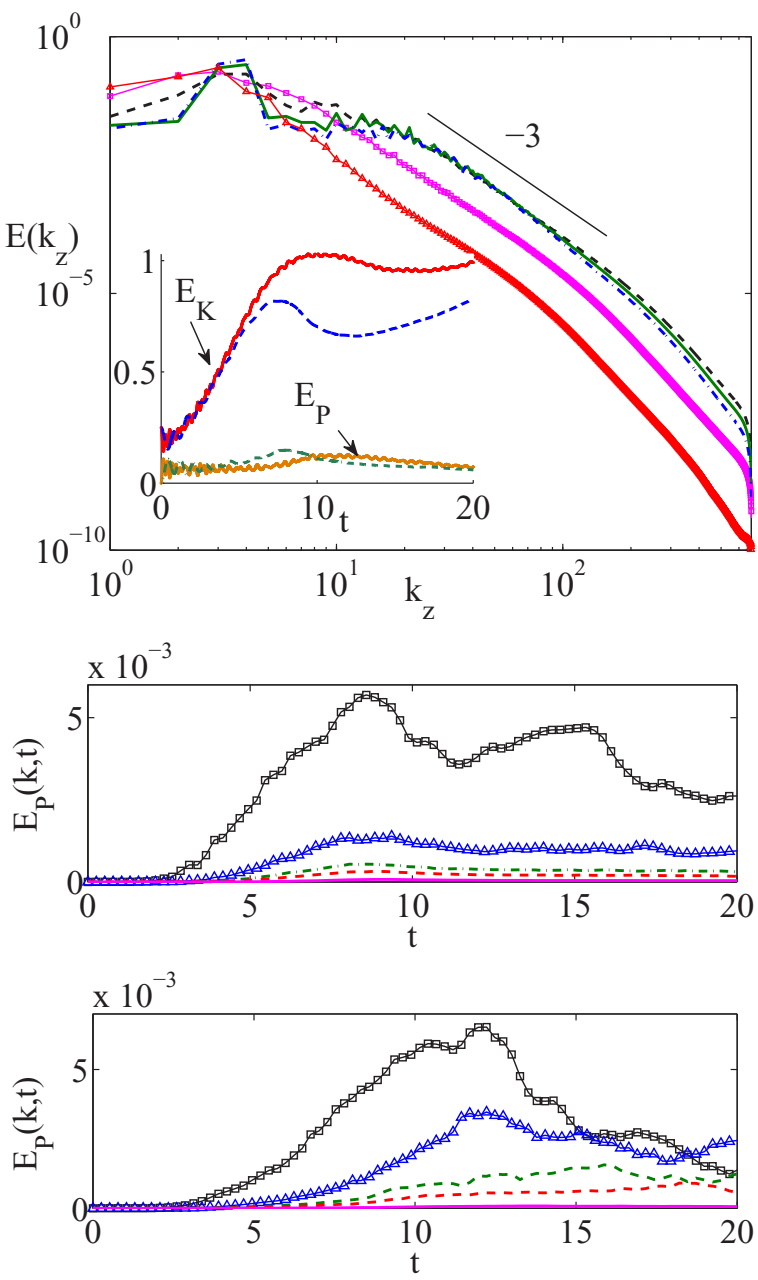

FIG. 2. (Color online) The top plot shows the parallel total energy spectrum for the run with $N=12$. Times correspond to $t=4.94$ (triangles, red), $t=7.54$ (squares, magenta), and $t>12$ (the remaining curves), when small scales have reached a turbulent steady state. $\mathrm{A} \sim k_{z}^{-3}$ scaling is shown as a reference. The inset shows the time evolution of the potential energy $E_{P}$ and of the kinetic energy $E_{K}$ in runs with $N=4$ (solid line) and $N=12$ (dashed line). Note the oscillations associated with internal gravity waves for $N=12$. The middle plot shows the time evolution of the potential energy for $k=10$ (squares, black), 20 (triangles, blue), 30 (dash-dotted, green), 40 (dashed, red), and 80 (solid, magenta) in the run with $N=4$. The bottom plot is the same as the middle but for the run with $N=12$. Note the larger fluctuations and bursts for large $k$ in this run reflected by the values of the standard deviation: $\sigma_{N=4}(k=10)=1.0 \times 10^{-3}$ and $\sigma_{N=12}(k=10)=8.4 \times 10^{-4}$, whereas, for larger values of $k$, $\sigma_{N=4}(k=40)=4.2 \times 10^{-5}$ and $\sigma_{N=12}(k=40)=1.1 \times 10^{-4}$.

for nonstratified flows with a passive scalar, and Ref. [21], which presents a model for rotating flows that display less extreme events than isotropic homogeneous turbulence. This latter model is also more complex in the sense that it takes into account the angle between the directional field variation and the direction of the imposed rotation. It leads to very good agreement between the model, experiments, and numerical simulations in terms of the lesser degree of intermittency in rotating flows, although positive skewness is observed in the horizontal plane linked to the inverse cascade of energy in these quasi-two-dimensional flows, with a nonmonotonic dependence on the Rossby number. Note also that this kind of model can be extended to consider the effects of shear in the flow (see, e.g., [22]); it is easy to see that in our model shear results in a stronger amplification of velocity variations.

The runaway toward strong gradients in Fig. 1 can be interpreted somewhat differently: For a given level of stratification as measured by $N$, there exists a scale $\ell$ at which strong negative tails in the velocity fluctuations will occur. For $N$ not too large, extreme events can develop even at large scale, where waves prevail, and thus be visible in the PDFs of the fields themselves. This behavior could be associated with the phenomenon of nonlinear amplification of waves observed in the nocturnal PBL [12,13], which could be directly related to the destabilization of vertical layers and in flows with internal shear and density fluctuations, as in the solar wind [9] (see also [23] for a similar solar wind observation of fat tails, but this time in the magnetic induction).

The model presented in this section is quite simple and similar models have proven useful in the analysis of turbulent flows, in the occurrence of quasisingular structures in the homogeneous isotropic case, and in the development of positive skewness of cyclonic vortices in rotating flows. In the next section we study the occurrence of extreme events in direct numerical simulations of fully developed turbulence using the Boussinesq equations.

\section{NUMERICAL SIMULATIONS}

\section{A. Description of the simulations}

We now examine the dynamics of stratified turbulence using direct numerical simulations (DNSs) at high resolution. To sustain the flow in a turbulent state, we use a three-dimensional large-scale random isotropic forcing on the right-hand side of Eq. (1). The forcing is applied in Fourier shells with wave numbers $k=2$ and 3 (i.e., the forced wave vectors $\mathbf{k}_{F}$ are such that $\left|\mathbf{k}_{F}\right| \in[2,3]$ ). Equations (1) and (2) are solved on grids of $2048^{3}$ points with the pseudospectral geophysical high-order suite for turbulence (GHOST) code, which is parallelized with hybrid MPI-OpenMP programming and has been tested on over 98000 compute cores [24]. As the amplification in the simplified model above can happen within the fluid, as opposed to near the boundaries, we consider for simplicity triperiodic boundary conditions within a $[0,2 \pi]^{3}$ box, with a second-order explicit Runge-Kutta temporal scheme and with dealiasing using a standard $2 / 3$ rule.

Two simulations were conducted for over 20 turnover times $\tau_{N L}=U / L$. In both simulations, the flow was started from random Gaussian isotropic initial conditions for the velocity and with no temperature fluctuations $(\theta=0$ at $t=0)$. The viscosity is such that the Reynolds number (the ratio of nonlinear to viscous effects) is $\operatorname{Re} \approx 2.5 \times 10^{4}$ for both runs. The simulations have either $\mathrm{Fr} \approx 0.1(N=4)$ or $\mathrm{Fr} \approx 0.03$ $(N=12)$. The buoyancy Reynolds number $R_{B}=\operatorname{ReFr}^{2}$ is found to be 250 and 22, respectively. Data for the statistical analysis are extracted after the peak of dissipation is reached and in the turbulent steady state of the flow. 


\section{B. Overall statistics}

Figure 2 gives the total energy spectrum in terms of vertical wave number when $N=12$ for different times; it is found to be compatible, after the peak of dissipation, with a $\sim k_{z}^{-3}$ scaling for wave numbers larger than $\approx 20$, whereas at larger scales a flat spectrum is obtained, as found in other studies [25,26]. This flat spectrum is associated with the accumulation of stratified layers in the vertical and ends for wave numbers larger than the so-called buoyancy wave number $k_{B} \sim N / U \approx 15$ for $U \approx$ 0.8 . This characteristic scale is thought to be of the order of the thickness of the stratified layers in the vertical direction (see, e.g., [25]).

In Fig. 2 we also show the time evolution of the kinetic and potential energies in both runs, defined here as $E_{K}=\left\langle|\mathbf{u}|^{2}\right\rangle$ and $E_{P}=\left\langle\theta^{2}\right\rangle$, respectively. The run with stronger stratification shows oscillations associated with internal gravity waves. The time evolution of the potential energy at different wave numbers is given in the middle and bottom panels of Fig. 2. At the smallest scales (small $\ell \sim 1 / k$, i.e., larger wave numbers), the time series of the run with $N=12$ is more bursty than for the run with $N=4$. By measuring the standard deviation of the time series, we verified that the time series at large scales in the run with $N=4$ have larger fluctuations than for $N=12$ (i.e., stratification smooths the evolution for sufficiently large $\ell$ ), while at small scales the opposite happens, in agreement with the qualitative behavior observed in the model. In particular, note that at $k=30$ and 40, the potential energy as a function of time is almost constant after $t \approx 10$ in the run with $N=4$, while it shows bursts and fluctuations in the run with $N=12$. In other words, there is measurably more excitation at small scale in the potential energy for the higher value of $N$.

\section{Extreme values and internal intermittency}

We examine now more closely the occurrence of extreme events in these high-resolution runs. As stratification increases, the flow is expected to become more stable and predictable, developing weaker events in the velocity and temperature. However, the opposite is observed. A better quantification of the strength of these events can be obtained from spatial information.

In Fig. 3 we show the PDFs for vertical velocity and temperature fluctuations and their vertical gradients for one snapshot of the fields in a time shortly after the peak of dissipation. We observe that (i) for a given field, the more stratified case is more bursty, as illustrated by the heavy tails of the histograms, which indicate a larger probability of the fields taking extreme values; (ii) the vertical velocity can take larger extreme values than the temperature; and conversely, (iii) the spatial derivative of the temperature takes larger extreme values than the derivative of the vertical velocity. Although non-Gaussian tails have been reported in PDFs of the field gradients of stratified flows, both numerically [27] as well as observationally [28,29], note that here the PDFs of the fields themselves are non-Gaussian, with the pointwise temperature and vertical velocity taking extreme values.

The non-Gaussianity in the PDFs of the field gradients is often associated with the usual internal intermittency observed in turbulent flows. However, the non-Gaussian tails in the PDFs of $\theta$ and $w$ indicate the development of extreme values in the
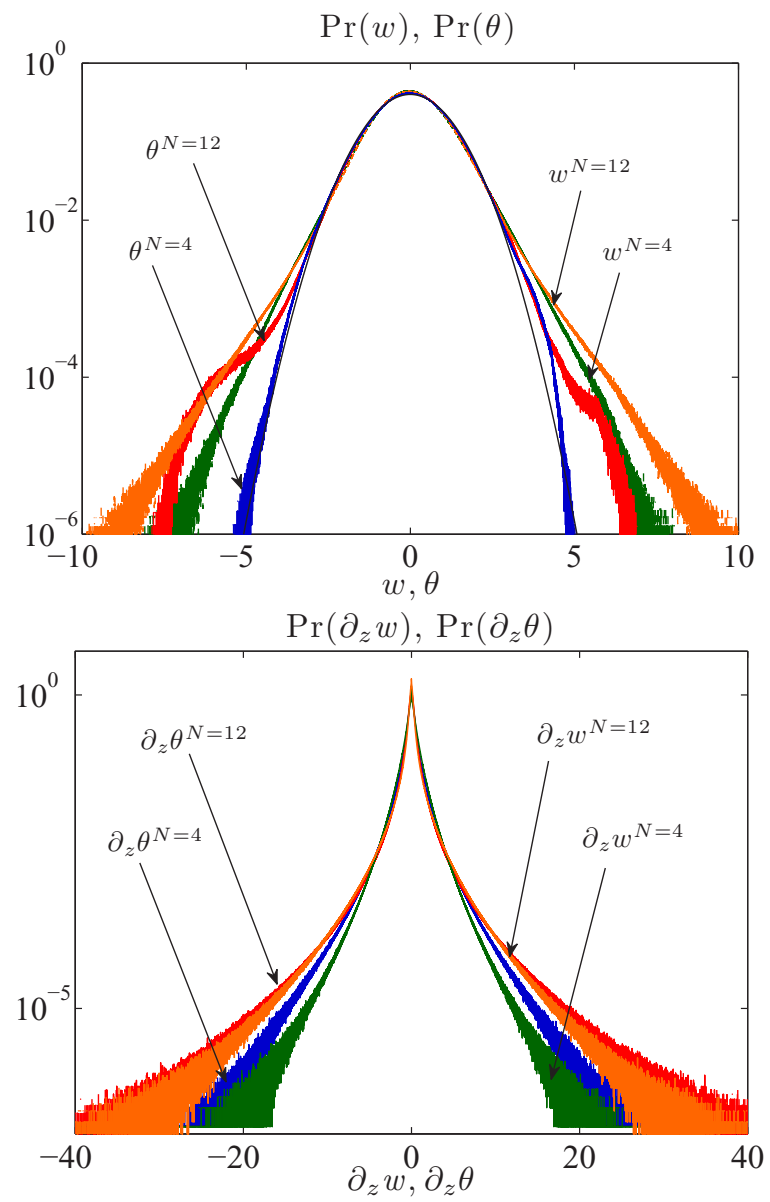

FIG. 3. (Color online) The top plot shows normalized histograms (in semilogarithmic coordinates) evaluated shortly after the peak of dissipation, for the temperature fluctuations $\theta$ and for the vertical component of the velocity $w$ for high-resolution simulations of a stratified flow with Froude number $\mathrm{Fr} \approx 0.1(N=4)$ and $\mathrm{Fr} \approx 0.03$ $(N=12)$. A normal distribution is shown (inner black curve) as a reference. The bottom plot shows PDFs of vertical derivatives for the same quantities. In all cases, the more strongly stratified flow with $N=12$ has larger probability of developing extreme events, as illustrated by the wider wings in the PDFs. For the fields themselves, the velocity has stronger tails than the temperature and the converse is true for their vertical derivatives.

amplitudes of the fields. These values are less extreme for the fields than for their gradients: At a probability of $10^{-5}$, one can observe gradients $\approx 20$ times the mean at this value of Reynolds number, but for the velocity one obtains a more modest fivefold increase, which nevertheless represents a huge acceleration of the flow. Such an increase is compatible with results from numerical simulations of frontal dynamics in the framework of the California current system [30].

Note also that the PDFs of the fields display asymmetries and bumps in the tails that may be the result of transient phenomena right after the peak of dissipation is reached. To verify this and improve statistical convergence of the data, we studied the PDFs at different times and computed PDFs of $\theta$ and $w$ integrated over three snapshots of the fields between $t \approx 10$ and 16 . The individual PDFs are similar to those 

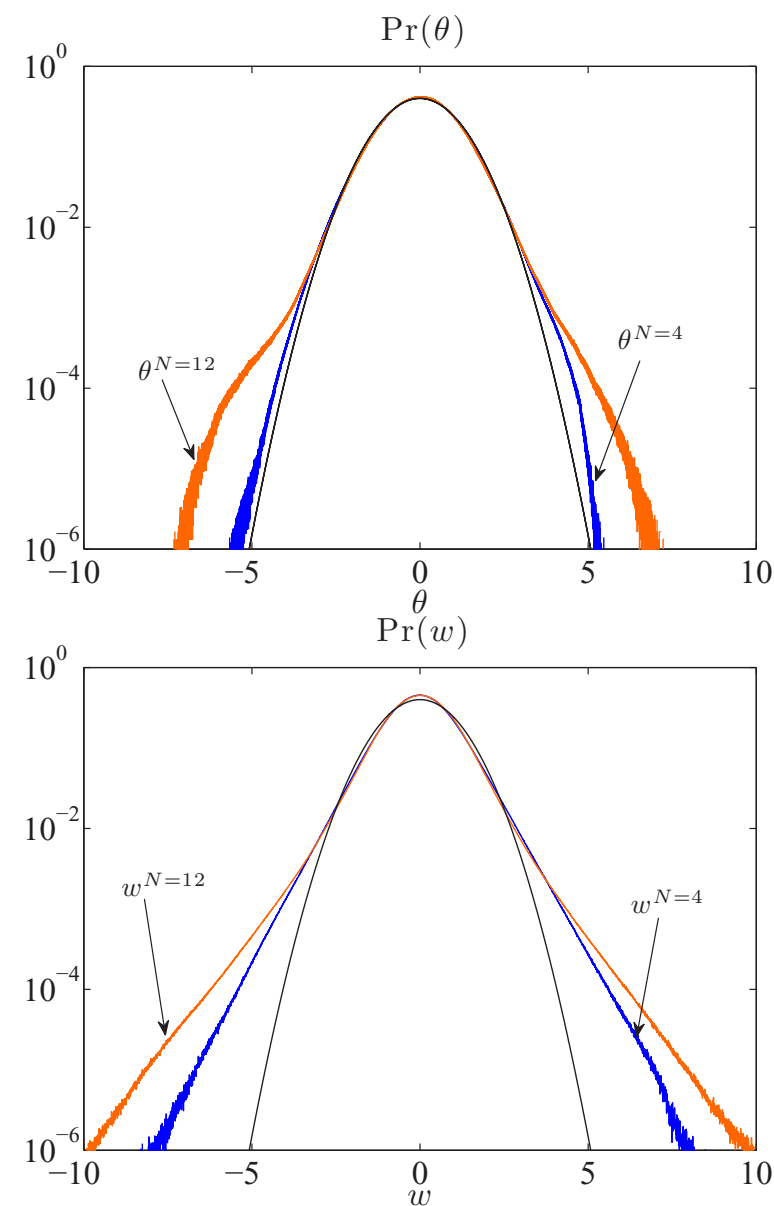

FIG. 4. (Color online) The top plot shows integrated in time PDFs (in semilogarithmic coordinates) of the temperature $\theta$ for three snapshots after the peak of enstrophy and for the simulations with Froude number Fr $\approx 0.1(N=4)$ and $\mathrm{Fr} \approx 0.03(N=12)$. The bottom plot is the same but for the vertical velocity $w$. In both panels, the inner black solid line corresponds to a normal distribution.

shown in Fig. 3. In Fig. 4 the integrated in time PDFs for $\theta$ and $w$ are shown. Each of these PDFs was constructed using $\approx 2.5 \times 10^{10}$ data points and the tails of the PDFs are better defined. The more stratified case is still more bursty, with large values for both the vertical velocity and the temperature. Also, the asymmetry and bumps in the PDF of the temperature still persists after the time integration.

Unlike the unusual behavior found in $\theta$ and $w$, the two horizontal components of the velocity field behave as expected and as observed in homogeneous isotropic turbulence. This can be seen in Fig. 5, which shows the PDFs of the perpendicular velocity $v_{\perp}$, which do not display strong tails. The curves are close to Gaussian, although the tails have slightly smaller amplitude than a Gaussian with the same dispersion.

\section{Accumulated moments}

In order to verify the convergence of the PDFs in the previous section, we also computed the accumulated moments

$$
C_{p}(\bar{x})=\int_{0}^{\bar{x}}|x|^{p} \operatorname{Pr}(x) d x,
$$

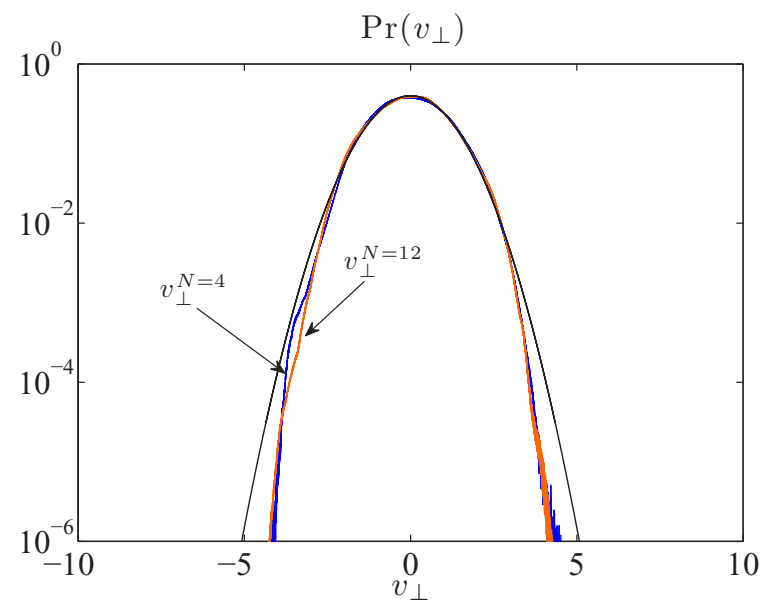

FIG. 5. (Color online) Integrated in time PDFs (in semilogarithmic coordinates) of the perpendicular velocity $v_{\perp}$ in the simulations with Froude number $\mathrm{Fr} \approx 0.1(N=4)$ and $\mathrm{Fr} \approx 0.03(N=12)$. As a reference, the outer black curve corresponds to a normal distribution.

where $x$ can be $u, v, w$, or $\theta$ and $\operatorname{Pr}(x)$ is the probability density function of the field $x$. As discussed, for example, in Ref. [31], the convergence of these moments with increasing values of $x$ gives an indication of the highest order $p$ for which moments of the PDF can be computed with good statistics.
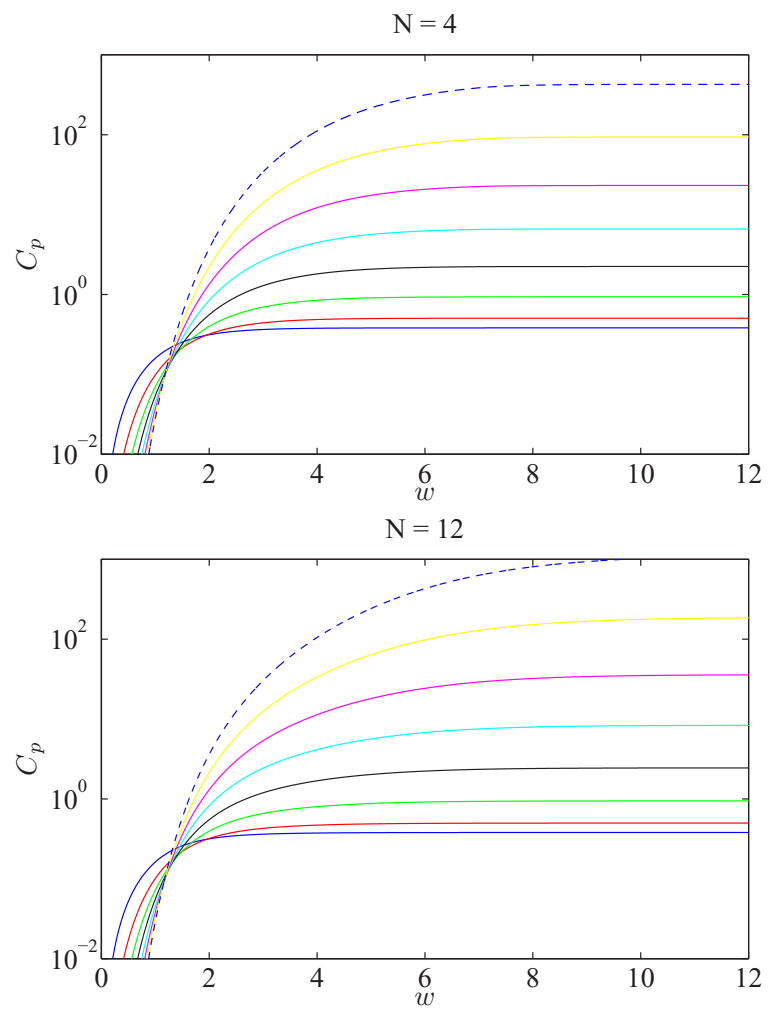

FIG. 6. (Color online) The top plot shows accumulated moments $C_{p}(w)$ for the vertical velocity in the simulation with $\mathrm{Fr} \approx 0.1(N=$ 4 ). The curves, from top to bottom, correspond to moments of order $p$ from 1 to 8 . The bottom plot is the same but for the simulation with Fr $\approx 0.03(N=12)$. 



FIG. 7. Shown above the grayscale bar are two-dimensional cuts in a $[0,2 \pi]^{3}$ box in the $x$ and $z$ directions for the flow with $\mathrm{Fr} \approx 0.1$ ( $N=4$, top row) and $\mathrm{Fr} \approx 0.03(N=12$, bottom row). The white segment is of unit length. The left-hand column corresponds to the temperature and the right-hand column to the vertical velocity. Note the strata in the temperature (the more strata, the higher the value of $N)$. For Fr $\approx 0.03$, sporadic overturning in the vertical cut is clearly visible. The flow with stronger stratification is less complex, but extreme values of the fields and their gradients are higher, leading to the development of turbulent bursts and localized mixing. Shown below the grayscale bar are details of some extreme events: KelvinHelmholtz $(\mathrm{KH})$ instability in the velocity field for $\mathrm{Fr} \approx 0.03(N=$ 12 , top), eddies in the temperature field for $\mathrm{Fr} \approx 0.1(N=4$, bottom left), and $\mathrm{KH}$ instability in the temperature field for $N=12$ (bottom right).

Figure 6 shows the accumulated moments for the vertical velocity $w$ in the runs with $N=4$ and 12, using the integrated in time PDFs. The vertical velocity has the PDF that shows the slowest convergence of high-order moments; all other fields show a faster convergence up to $p=8$. Good convergence is observed up to eighth order in the run with $N=4$, while convergence in the $N=12$ run is only marginal for the highest moment, with the rest of the moments showing better convergence.

\section{E. Intermittent structures}

Figure 7 displays vertical slices of temperature and vertical velocity for the same two flows. As expected, in the less

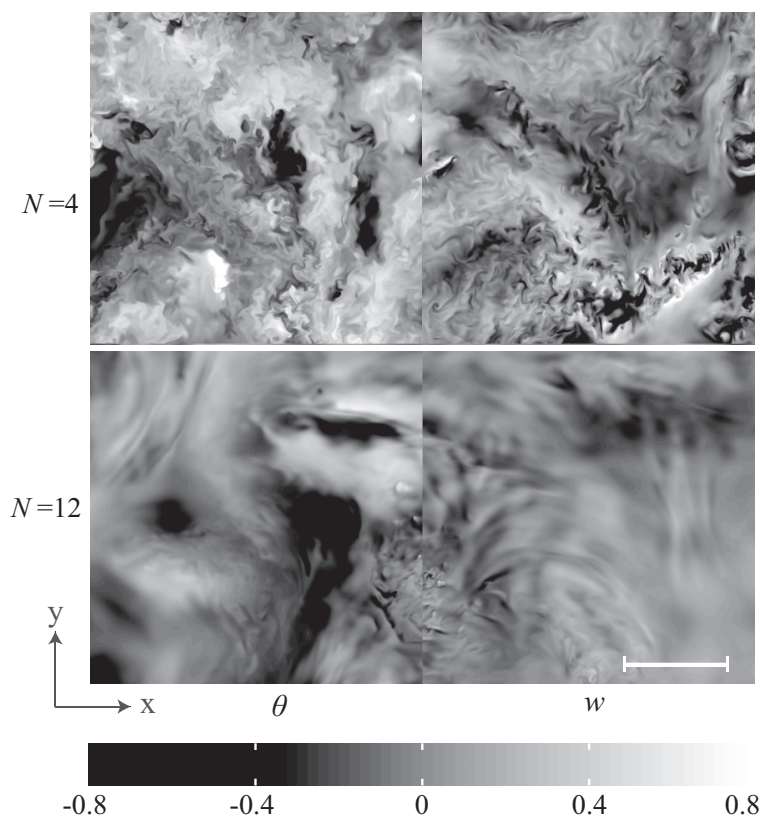

FIG. 8. Two-dimensional cuts in a $[0,2 \pi]^{3}$ box in the $x$ and $y$ (horizontal) directions for the flow with $\mathrm{Fr} \approx 0.1(N=4$, top row) and $\mathrm{Fr} \approx 0.03$ ( $N=12$, bottom row). The white segment is of unit length. The left-hand column corresponds to the temperature and the right-hand column to the vertical velocity. Note the stripes in the fields in the run with $N=12$, associated with the overturning events in Fig. 7, this time shown from the top.

stratified flow, turbulence in the form of multiple eddies is ubiquitous. The more stratified flow seems more ordered, with multiple layers and strong vertical gradients. Moreover, strong small-scale Kelvin-Helmholtz-type instabilities develop and dominate the small-scale dynamics as seen in several locations (see Fig. 7); in this flow, turbulence comes in localized bursts with strong values of the fields. Figure 7 indicates that the maxima of the vertical velocity and temperature take place in the Kelvin-Helmholtz overturning events, suggesting that the extreme values in the PDF of $\theta$ and $w$ are associated with these instabilities. On the other hand, maxima of the temperature gradients take place in the sharp fronts that are known to develop in stratified turbulence.

Note also the layered structures in the vertical, especially in the temperature. The number of layers depends on the stratification, with vertical correlation lengths $\ell_{b}$ of $\approx 1 / 8$ of the box in the flow with $\mathrm{Fr} \approx 0.1(N=4)$ and $\approx 1 / 26$ of the box for $\mathrm{Fr} \approx 0.03(N=12)$. If these values are used for $\ell$ in the model given by Eqs. (4) and (5) together with the corresponding values of $N$, a faster growth of the fields is obtained for the linearly more stable flow, in good agreement with the DNSs and as indicated by observations. The vertical correlation length is defined as

$$
\ell_{b}=2 \pi \frac{\int E\left(k_{z}\right) d k_{z}}{\int k_{z} E\left(k_{z}\right) d k_{z}},
$$

where $E\left(k_{z}\right)$ is the total energy parallel spectrum.

Figure 8 shows a similar visualization of the temperature and the vertical velocity for both flows, but this time with a cut in the $(x, y)$ plane. In the simulation with $N=12$, stripes 
can be seen in both fields. These stripes are associated with the overturning events in Fig. 7, but this time shown from the top.

\section{CONCLUSION}

\section{A. Summary of results}

The main result of this paper is the occurrence of nonGaussian wings in the probability distribution functions of vertical velocity and temperature fluctuations in strongly stratified turbulence in the Boussinesq framework, obtained by analyzing data from large direct numerical simulations on grids of $2048^{3}$ points. We also showed evidence that internal intermittency is larger in the simulation with stronger stratification, a flow that was instead expected to be more stable.

The extreme events in the fields, as well as the smallscale internal intermittency associated with the non-Gaussian wings in the field gradients, are compatible with a simplified model mimicking vertical variations of the vertical velocity and of temperature fluctuations in the spirit of singularity development in homogeneous isotropic turbulence [17] and of strong gradients in the vertical as predicted in Ref. [32]. As mentioned in the Introduction, such strong gradients have been observed in a variety of geophysical flows. Both our model and the computations point to stably stratified flows spontaneously developing long-lasting bursts that are stronger for stronger stratification, but possibly with a nonmonotonic behavior in terms of variation with Froude number, a known feature of stratified turbulence (see, e.g., $[33,34]$ and references therein).

We can infer from our model, as well as from the spatial visualizations of the fields, that propagating gravity waves are nonlinearly amplified, resulting in their breaking and the generation of turbulence when the linear and nonlinear effects are balanced. The nonlinear tendency to develop negative gradients occurs at all scales, including large scales. This would explain why non-Gaussian statistics are observed even in the velocity and temperature fields. Although the model presented here is very simple, it correctly captures that only the temperature and the vertical velocity display extreme values, associated with their coupling through gravity waves; indeed, gravity acts only in the vertical and the horizontal velocity will be only affected by it through its nonlinear coupling to the vertical velocity by the advection term (and pressure). Our simple model is also compatible with the so-called saturation spectrum $E\left(k_{z}\right) \sim N^{2} k_{z}^{-3}$, which is broadly observed in the atmosphere and the oceans, where such strong bursty events are also commonly observed. Note that for $E\left(k_{z}\right) \sim N^{2} k_{z}^{-3}$, the eddy turnover time is proportional to $1 / N$, validating the hypothesized dynamical balance across all scales [32,35].

One central tenet of this dynamical balance developed first in Ref. [32] is that the Froude number based on a vertical length scale, the so-called buoyancy length scale $L_{B} \sim 1 / k_{B}$, is of order unity however small the global Froude number of the flow is. This implies that turbulence must be strong in the vertical for strongly stratified flows, provided some nonlinear interactions can develop. In that case, it seems plausible that a model based on the development of strong fields with a quadratic nonlinearity but including the effect of oscillatory motions should capture the fundamental behavior of such flows.

\section{B. Discussion}

It is known that waves in turbulence can lead to intermittency, such as with rogue waves in the ocean; anomalous concentrations of particles in large-scale waves [36] and enhancement of non-Gaussian initial conditions [37] have been observed as well. In a turbulent flow, internal intermittency has also been related to a global correlation between interacting scales and as such may be indicative of a system close to criticality [38]. Similarly, nonlocality of interactions between small-scale and large-scale eddies is advocated in Ref. [39] as directly related to the bursting phenomenon of the nocturnal PBL. Our model indicates a different origin for the strong localized events, associated with a positive feedback in the vertical between nonlinear steepening and wave motions, and is consistent with the simulations that indicate that a more stably stratified flow has stronger bursts.

Of course, other mechanisms could be envisaged for our result of extreme events in the fields and in the field gradients. A possible example is a parametric instability, the forcing being provided by a spectrum of nonlinear eddies which arise from nonlinear coupling and can have frequencies comparable to the imposed Brunt-Väisälä frequency $N$ due to stratification. Note that such an explanation leads us again to Froude numbers based on vertical length scales of order one, as discussed above in the context of the model, as the Froude number can be interpreted as the ratio of the wave period to the turnover time.

Another explanation for intermittency in strongly stratified flows may be linked to the phenomenon of on-off intermittency [40]. In that case, one can envisage two quasi-invariant subspaces for the dynamics, say, two quasigeostrophic layers, which are nevertheless slightly coupled through vertical gradients. As the respective representative points of these two subsystems become closer in phase space, strong interactions develop for a few characteristic times before the quasilinear behavior is recovered. Our model does not preclude these effects from playing a role. However, note that in the cases of parametric instability, on-off intermittency, and subcritical transitions, the systems that are often envisaged are quite well ordered with only a few spatial modes excited. In our case, the simulations have a well developed inertial range spectrum and the extreme events develop in the turbulent steady state.

In particular, the study of subcritical transitions in a variety of classical configurations such as Taylor-Couette or shear (pipe) flows is well developed (see, e.g., [10,11] for reviews). They tend to appear at lower Reynolds number than supercritical ones, due to the effect of nonlinear coupling. The coexistence of structures implies the existence of fronts as barriers between the quiescent and active regions of the flow undergoing the transition. These problems can, e.g., be studied by analogy to percolation (between quiet and active sites) [41]. Intermittency and hysteresis are systematically invoked in these transitions and may be linked to the sharp boundaries between laminar and turbulent spots, identified early on as spiral bands [42]. However, fat wings in the PDFs of the 
velocity have not been identified in the specific context of subcritical transitions.

From the model and the simulations, we thus conclude that the intermittent bursts in our case are associated with the direct coupling between vertical velocity and temperature fluctuations, providing a possible explanation for the intriguing observation of strong intermittency in the more stably stratified nocturnal planetary boundary layer. This study may thus lead to more useful parametrizations of stably stratified flows in weather and climate models by formulating a stochastic eddy noise [43] that explicitly incorporates the nonlinear coupling described herein (for a quasinormal closure, see [44]). In the context of strongly stratified turbulence, a path was recently described in detail between the large scales in quasigeostrophic equilibrium and the small scales that provide the dissipation, the link between the two scales being provided by the existence of fronts that are reminiscent of the passive tracer case [45]. It was also shown in Ref. [46] that such flows, in the presence of moderate rotation, can display both a direct and an inverse cascade of energy, both with a constant flux. The role of these effects in the flow intermittency is left for future studies.

\section{ACKNOWLEDGMENTS}

This work was supported by NSF/CMG Grant No. 1025183. C.R. acknowledges support from two RSVP/CISL grants. Computer time on Yellowstone through ASD was provided by NCAR under sponsorship of NSF. A.P. gratefully acknowledges financial support from LASP. A.P. also acknowledges useful discussions with the WINABL group early on in this work and in particular with J. Finnigan and P. Sullivan. C.R., P.D.M., and A.P. gratefully acknowledge useful discussions with R. Marino.
[1] T. Antal, M. Droz, G. Györgyi, and Z. Ràcz, Phys. Rev. Lett. 87, 240601 (2001).

[2] S. T. Bramwell, K. Christensen, J.-Y. Fortin, P. C. W. Holdsworth, H. J. Jensen, S. Lise, J. M. Lopez, M. Nicodemi, J.-F. Pinton, and M. Sellitto, Phys. Rev. Lett. 84, 3744 (2000).

[3] A. Pumir, Phys. Fluids 8, 3112 (1996).

[4] M. S. Paoletti, M. E. Fisher, K. R. Sreenivasan, and D. P. Lathrop, Phys. Rev. Lett. 101, 154501 (2008).

[5] A. W. Baggaley and C. F. Barenghi, Phys. Rev. E 84, 067301 (2011).

[6] X. Capet, J. McWilliams, M. Molemaker, and A. Shchepetkin, J. Phys. Oceanogr. 38, 29 (2008).

[7] H. L. Liu, Geophys. Res. Lett. 34, L08815 (2007).

[8] R. Marino, L. Sorriso-Valvo, V. Carbone, P. Veltri, A. Noullez, and R. Bruno, Planet. Space Sci. 59, 592 (2011).

[9] R. Marino, L. Sorriso-Valvo, R. D’Amicis, V. Carbone, R. Bruno, and P. Veltri, Astrophys. J. 750, 41 (2012).

[10] P. Manneville, Dissipative Structures and Weak Turbulence (Academic, Boston, 1990).

[11] B. Eckhardt, T. Schneider, B. Hof, and J. Westerweel, Annu. Rev. Fluid Mech. 39, 447 (2007).

[12] J. Sun, L. Mahrt, R. Banta, and Y. Pichugina, J. Atmos. Sci. 69, 338 (2012).

[13] J. Finnigan, Bound. Layer Meteorol. 90, 529 (1999).

[14] B. J. H. V. de Wiel, A. Moene, and H. Jonker, J. Atmos. Sci. 69, 3097 (2012).

[15] C. Drüe and G. Heinemann, Bound. Layer Meteorol. 124, 361 (2007).

[16] B. Stevens and G. Feingold, Nature (London) 461, 607 (2009).

[17] P. Vieillefosse, J. Phys. 43, 837 (1982).

[18] C. Meneveau, Annu. Rev. Fluid Mech. 43, 219 (2011).

[19] K. Polzin and Y. Lvov, Rev. Geophys. 49, RG4003 (2011).

[20] Y. Li and C. Meneveau, J. Fluid Mech. 558, 133 (2006).

[21] Y. Li, Physica D 239, 1948 (2010).

[22] M. Chong, J. Soria, A. Perry, J. Chacin, B. Cantwell, and Y. Nas, J. Fluid Mech. 357, 225 (1998).
[23] J. M. Weygand, M. G. Kivelson, K. K. Khurana, H. K. Schwarzl, S. M. Thompson, R. L. McPherron, A. Balogh, L. M. Kistler, M. L. Goldstein, J. Borovsky, and D. A. Roberts, J. Geophys. Res. 110, A01205 (2005).

[24] P. Mininni, D. Rosenberg, R. Reddy, and A. Pouquet, Parallel Comput. 37, 316 (2011).

[25] Y. Kimura and J. R. Herring, J. Fluid Mech. 698, 19 (2012).

[26] C. Rorai, D. Rosenberg, A. Pouquet, and P. D. Mininni, Phys. Rev. E 87, 063007 (2013).

[27] G. Brethouwer, P. Billant, E. Lindborg, and J.-M. Chomaz, J. Fluid Mech. 585, 343 (2007).

[28] V. Petoukhov, A. V. Eliseev, R. Klein, and H. Oesterle, Tellus 60A, 11 (2008).

[29] D. H. Lenschow, M. Lothon, S. D. Mayor, P. P. Sullivan, and G. Canut, Bound. Layer Meteorol. 143, 107 (2012).

[30] X. Capet, J. McWilliams, M. Molemaker, and A. Shchepetkin, J. Phys. Oceanogr. 38, 44 (2008).

[31] T. Gotoh, D. Fukayama, and T. Nakano, Phys. Fluids 14, 1065 (2002).

[32] P. Billant and J.-M. Chomaz, Phys. Fluids 13, 1645 (2001).

[33] P. Bartello and S. Tobias, J. Fluid Mech. 725, 1 (2013).

[34] A. Mashayek, C. P. Caullfield, and W. R. Peltier, J. Fluid Mech. 736, 570 (2013).

[35] E. Lindborg, J. Fluid Mech. 550, 207 (2006).

[36] G. Falkovich and A. Fouxon, Phys. Rev. Lett. 94, 214502 (2005).

[37] Y. V. Lvov and S. Nazarenko, Phys. Rev. E 69, 066608 (2004).

[38] B. Portelli, P. C. W. Holdsworth, and J.-F. Pinton, Phys. Rev. Lett. 90, 104501 (2003).

[39] D. C. Fritts, L. Wang, and J. Werne, Geophys. Res. Lett. 36, 396 (2009).

[40] Y. Pomeau and P. Manneville, J. Phys. 41, 1235 (1980).

[41] M. Sipos and N. Goldenfeld, Phys. Rev. E 84, 035304(R) (2011).

[42] D. Coles, J. Fluid Mech. 21, 385 (1965).

[43] T. N. Palmer, Q. J. R. Meteorol. Soc. 138, 841 (2012).

[44] V. M. Gryanik, J. Hartmann, S. Raasch, and M. Schröter, J. Atmos. Sci. 62, 2632 (2005).

[45] M. Molemaker, J. McWilliams, and X. Capet, J. Fluid Mech. 654, 35 (2010).

[46] A. Pouquet and R. Marino, Phys. Rev. Lett. 111, 234501 (2013). 